\title{
Intestinal obstruction in an adolescent female
}

Institute of Medical Sciences, Banaras Hindu University, Varanasi 221005, India

Department of Surgery

A Kumar

A Gautam

Department of

Pathology

S Chaturvedi

S Gupta

Correspondence to

Dr A Gautam

Accepted 28 June 1995

\author{
Anand Kumar, Amitabh Gautam, Sunanda Chaturvedi, Saroj Gupta
}

A 16-year-old girl was admitted with a history of abdominal pain, recurrent vomiting and abdominal distension for the last three years. On examination there was a diffuse lump occupying the central abdomen. Sigmoidoscopy showed normal mucosa till $20 \mathrm{~cm}$. Apart from a polymorphonuclear leucocytosis (total white cell count of $25 \times 10^{9} / 1$ with $80 \%$ polymorphs), other laboratory investigations were within normal limits. A barium enema revealed incomplete filling of the colon proximal to the rectosigmoid junction. A laparotomy, with a provisional diagnosis of subacute intestinal obstruction, was undertaken. The entire small intestine, caecum and colon up to the rectosigmoid junction were found to be encased in a thick fibrous membrane. This membrane was completely excised (figure 1) and lysis of the inter-loop adhesions was performed. The postoperative recovery was uneventful. 2.

A photomicrograph of a $\mathrm{H} \&$ E-stained section from the excised membrane is shown in figure
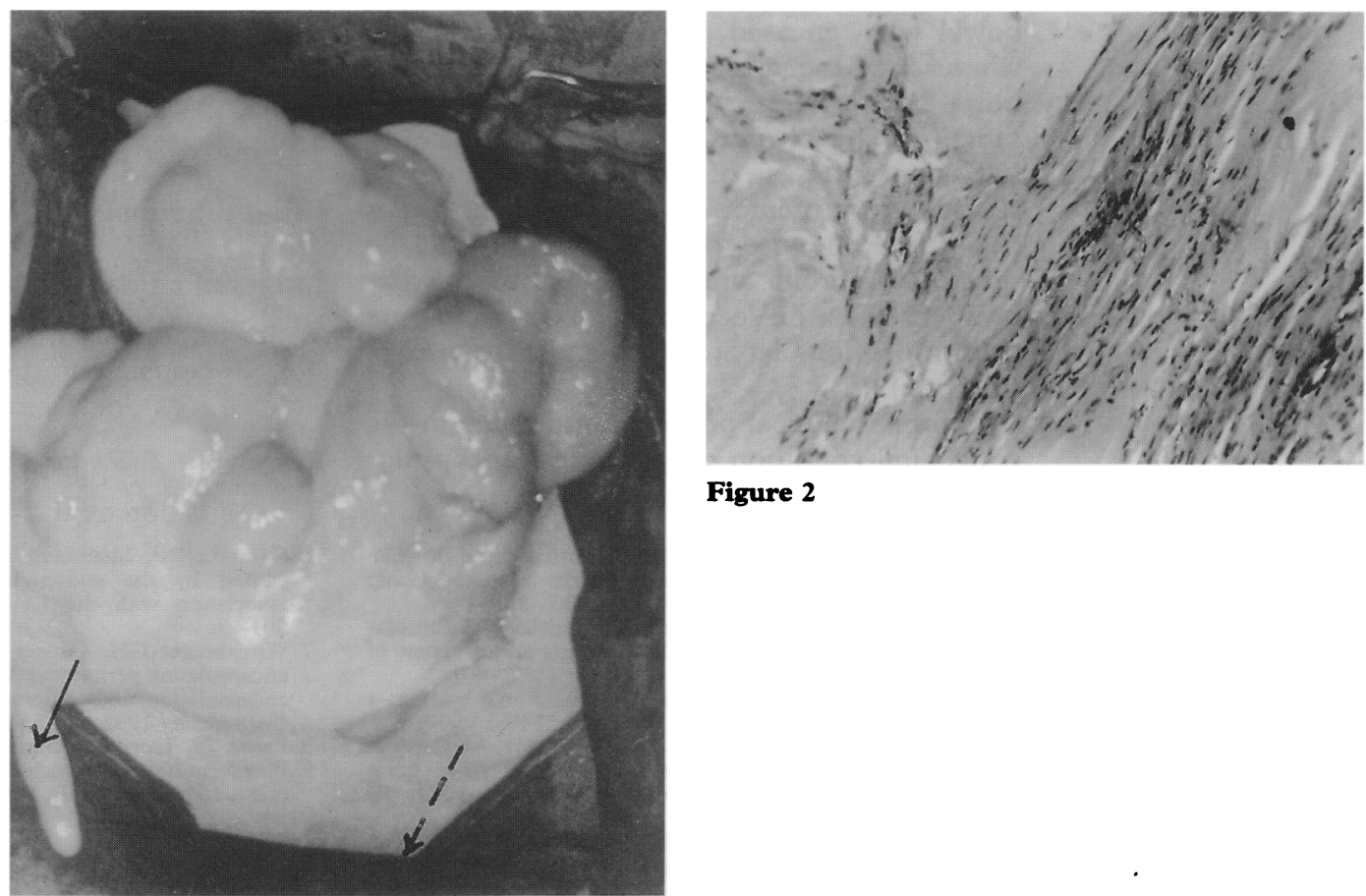

Figure 2

Figure 1

Question

What is this condition called? 


\section{Answer}

Abdominal cocoon. Histological examination of the excised cocoon wall, showed characteristic neurofibromatous components seen as twisted wire-like neurofibrils (figure 2). Interspersed in between were variable amounts of collagen bundles.

Abdominal cocoon, or small bowel obstruction due to a membranous encasement, is a rare clinical entity. It has been described predominantly in adolescent females residing in tropical and subtropical areas. ${ }^{1,2}$ The aetiology of this condition remains obsure, although an infective pathology is suspected, ${ }^{1}$ due to retrograde peritonitis through the fallopian tube. Histological examination of the excised membrane usually reveals fibrous tissue only, with no evidence of inflammation or granuloma formation. ${ }^{1,3}$

Clinically, the present case had many similarities to previously described cases of abdominal cocoon. ${ }^{1,2}$ The patient was an adolescent female from a tropical area. However in the previously described cases, only the small bowel was encased, completely or partly, ${ }^{1}$ whereas, in the present case the entire small bowel and colon up to the rectosigmoid junction were involved.

Known causes of sclerosing peritonitis, resulting in a membranous encasement of the bowel are the use of $\beta$-blockers, especially practolol, ${ }^{4}$ chronic ambulatory peritoneal dialysis, ${ }^{5}$ use of a LeVeen shunt, ${ }^{6}$ talc granuloma from a previous laparotomy ${ }^{7}$ and liver cirroh$\operatorname{sis}^{3}$ (box).

A similar clinical presentation is seen in the congenital condition of 'peritoneal encapsula-

1 Macklin J, Hall C, Feldman MA. Unusual cause of small bowel obstruction in adolescent girls: the abdominal cocoon. $\mathcal{F} R$ Coll Surg Edinb 1991; 36: 50-2.

2 Sieck JO, Cowgill R, Larkworthy W. Peritoneal encapsulation and abdominal cocoon. Case reports and a review of the literature. Gastroenterology 1983; 84: 1597-601.

3 Seng LK, Mahadaven M, Musa A, Abdominal cocoon: a report of two cases. Br $\mathcal{Y}$ Surg 1993; 80: 1149.

4 Brown P, Baddeley H, Read AE, Davies JD, McCarry J.

Sclerosing peritonitis: an unusual reaction of a betaadrenergic blocking drug (practolol). Lancet 1974; ii: $1477-81$.

5 Bradely JA, McWhinnie DL, Hamilton DHN, et al.

Sclerosing obstructive peritonitis after continuous ambulatory peritoneal dialysis. Lancet 1983; ii: 113-4.
Differential diagnosis of membranous encasement of bowel

- abdominal coccon

- congenital peritoneal encapsulation

- sclerosing peritonitis: due to $\beta$-blockers, use of LeVeen shunt, talc granuloma from previous laparotomy, chronic ambulatory peritoneal dialysis, or in liver cirrhosis

- intra-abdominal plexiform neurofibroma (present case)

tion'.2 There is an accessory membrane, derived from the peritoneum of the yolk sac, ${ }^{1}$ present in front of the small bowel, which can be easily removed. However, this condition is completely asymptomatic and is found incidentally at laparotomy or autopsy.

Neurofibromas, though rare, are the most common tumours involving nerves. They may arise in any nerve containing Schwann cells. Intra-abdominal neurofibromas are characteristically retroperitoneal, although plexiform neurofibromas involving the intestinal mesentery and the liver have been described. ${ }^{8}$ However, to our knowledge, this is the first reported case of an intra-abdominal neurofibroma presenting as an abdominal cocoon.

\section{Final diagnosis}

Intra-abdominal neurofibroma presenting as an abdominal cocoon.

Keywords: abdominal cocoon, neurofibroma

6 Cambria RP, Shamberger RC. Small bowel obstruction caused by the abdominal cocoon syndrome: possible association with the LeVeen shunt. Surgery 1984; 95: $501-3$.

7 Wirnsberger GH, Ganser K, Domej W et al. Sclerosing encapsulating peritonitis: differential diagnosis to peritoneal encapsulation and abdominal cocoon - a case report. $Z$ Gastroenterol 1992; 30: 534 - 7 .

8 Partin JS, Lane BP, Partin JC, Edelstein LR, Priebe CJ. Plexiform neurofibromatosis of the liver and mesentery in a child. Hepatology 1990; 12: 559-64. 